\title{
ANÁLISIS DEL CARBONO SECUESTRADO EN HUMEDALES ALTOANDINOS DE DOS ÁREAS PROTEGIDAS DEL ECUADOR
}

\section{ANALYSIS OF CARBON SECUESTRATION IN TWO ANDEAN WETLAND PROTECTED AREAS ECUADOR}

\author{
David Suárez Duque ${ }^{1}$, Cristhian Acurio ${ }^{2}$, Segundo Chimbolema² y Ximena Aguirre $^{3}$
}

\begin{abstract}
Resumen
Los humedales son considerados globalmente importantes por almacenar carbono, debido a la alta productividad de las plantas y a la baja descomposición de la materia orgánica que ocurren en sus suelos inundados. En las áreas protegidas se está conservando y manejando ecosistemas para mantener en buen estado la biodiversidad y servicios ambientales; por ende, se contribuye al almacenamiento de carbono. El objetivo de este estudio fue analizar el potencial de carbono orgánico que se estaría almacenando en los humedales de altura de la Reserva Ecológica Antisana (REA) y el Parque Nacional Cajas (PNC) del Ecuador. La contribución de cada humedal a la captura de carbono orgánico fue determinada a través de un muestreo sistemático con trabajo de campo y laboratorio, en el que se analizó de cada área protegida tres humedales en diferente estado de conservación. En la REA y el PNC, el humedal considerado como “conservado” tiene el más alto contenido de carbono. Los humedales tienen un potencial de secuestro de carbono, pero el drenado, quemas y pastoreo provocan la liberación de $\mathrm{CO}_{2}$, por lo que la conservación de los humedales es un punto crítico en la regulación del ciclo de carbono en la tierra.
\end{abstract}

Palabras clave: Humedales, captura de carbono, páramos, suelo, servicios ambientales, cambio climático.

\begin{abstract}
Wetlands are considered globally important for storing carbon, due to the high plant productivity, and low decomposition of organic matter occurring in their flooded soils. Protected areas around the world are being conserved and ecosystems managed to maintain a good state of biodiversity and environmental services; therefore, contributing to carbon storage in these. The aim of this study was to analyze the potential of carbon that could be stored in the high wetlands of the Antisana Ecological Reserve (REA) and the Cajas National Park (PNC) of Ecuador. Wetland with different states of conservation were analyzed in each protected area; the contribution to organic carbon capture was determined through a systematic sampling with field and laboratory work. In the REA and the PNC, the wetland considered "preserved" had the highest organic carbon content. Wetlands have a significant potential for carbon sequestration, but drainage, drying, burning and grazing trigger the release of $\mathrm{CO}_{2}$, thus the conservation of wetlands is critical in regulating the carbon cycle on Earth.
\end{abstract}

Key words: Wetlands, carbon capture, moorlands, paramo, soil, ecosystem services, climate change.

\section{Introducción.}

En las cumbres de la cordillera de los Andes, por encima de la línea de bosque, se encuentran los páramos andinos, donde interaccionan suelo, clima, biota y humanos. La región de vida paramuna, según Rangel (2000), “comprende las extensas zonas que coronan las cordilleras entre el bosque andino y el límite inferior de las nieves perpetuas”. Desde hace varios años, se discute la importancia de los páramos, por su endemismo, paisaje, regulación hídrica, entre otros servicios ecosistémicos (Vega \& Martínez, 2000). A pesar de que el conocimiento de la dinámica de carbono en los ecosistemas altoandinos es aún limitado (Gibbon et al., 2010), los pocos estudios realizados estiman que el páramo tiene reservorios importantes de carbono (Podwojewski \& Poulenard, 2000 a, b), que pueden contribuir a la mitigación de los gases de efecto invernadero.

La baja temperatura en el páramo reduce dramáticamente las tasas de descomposición de materia orgánica, y el proceso se vuelve lento con un reducido stock de hojarasca y biomasa aérea (Hofstede, 1999). Los suelos en los altos Andes del Ecuador —Andisoles_, según Hofstede \& Aguirre 
(1999) “están formados por cenizas volcánicas jóvenes bajo condiciones frescas y húmedas; como resultado la descomposición de materia orgánica es baja”. A esto se suma la formación de complejos órgano-metálicos en el suelo, que contribuyen a una menor descomposición. Esto ocasiona que estos suelos tengan altos contenidos de materia orgánica, por lo tanto, una gran capacidad de retención de agua (Hofstede \& Aguirre, 1999). Estas condiciones de: alta humedad, bajas temperaturas, características especiales del suelo y la lenta descomposición de materia orgánica; posibilitan que el carbono se almacene en gran cantidad en los suelos paramunos (Hofstede, 1999).

Algunos de los humedales palustres que se encuentran en los páramos son conocidos como bofedales. Estos ecosistemas son áreas inundadas o seminundadas sobre suelos impermeables que atrapan el agua. Estas características edáficas especiales de los humedales, permite que crezca una rica vegetación en forma de almohadillas y, usualmente existe, materia vegetal muerta, que se transforma en turba (Izurieta, 2004). Los humedales son considerados globalmente importantes por almacenar carbono (Mistch \& Gosselink, 2000). Esto se debe a la alta productividad de las plantas y a la baja descomposición de la materia orgánica que ocurren en sus suelos inundados (Collins \& Kuehl, 2000). La acumulación de carbono en los humedales, según Hernández (2010) "se realiza en dos compartimientos, la biomasa vegetal y los suelos”.

En las áreas protegidas en todo el mundo se está conservando y manejando ecosistemas para mantener en buen estado su biodiversidad y servicios ambientales. Con ello se contribuye al almacenamiento de carbono y se evita las emisiones por deforestación y degradación (Busch \& Grantham, 2013). En el caso del Ecuador, el páramo es el ecosistema mejor representado en el sistema nacional de áreas protegidas (Cuesta et al., 2015). Los humedales (bofedales) de altura son ecosistemas importantes dentro de este mosaico paramuno. Por esta razón, los objetivos de este estudio fueron: determinar el potencial de carbono orgánico que estaría almacenando en los humedales palustres de altura de dos áreas protegidas que contienen páramo herbáceo en el Ecuador, y analizar la influencia del estado de conservación en la captura de carbono en estos humedales.

\section{Materiales y métodos.}

Área de estudio. La Reserva Ecológica Antisana y el Parque Nacional Cajas son áreas protegidas del Ecuador, se encuentran en la cordillera de los Andes y protegen una muestra importante de páramo herbáceo del país. La Reserva Ecológica Antisana (REA) se localiza en la cordillera oriental, en las provincias de Napo y Pichincha, al centro-norte del Ecuador (78 1'80” O; 0 34'80” S). Comprende los bosques andinos y páramos localizados en las planicies y estribaciones del volcán Antisana. El Parque Nacional Cajas (PNC) se ubica en la cordillera occidental, al sur de los Andes ecuatorianos (79¹4'09” O; $2^{\circ}$ 50'22” S); está situado al noroeste de la ciudad de Cuenca, provincia del Azuay, al sur del Ecuador (Figura 1), comprende páramos herbáceos, bosques alto andinos y bosques de Polylepsis del macizo del Cajas (MAE, 2013).

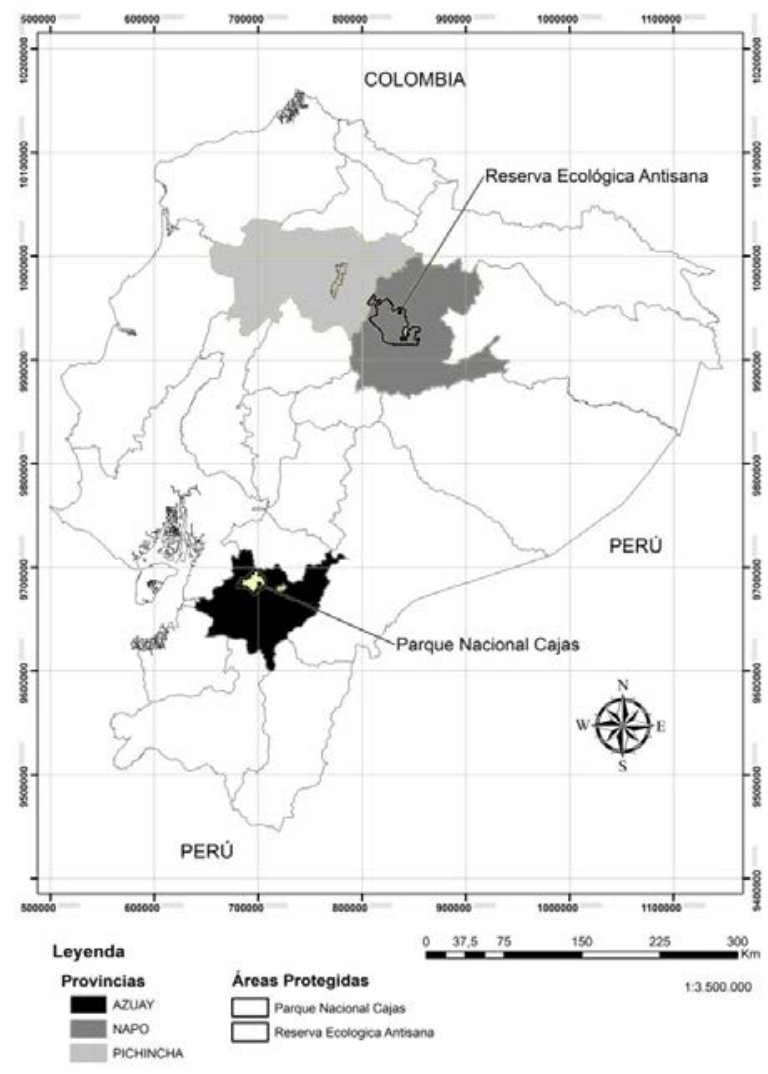

Figura 1. Ubicación de la Reserva Ecológica Antisana y Parque Nacional Cajas.

En el Parque Nacional Cajas y la Reserva Ecológica Antisana, los humedales, producen, almacenan y proveen agua para la ciudad de Cuenca y Quito, respectivamente. Pese a que las dos áreas protegidas son parte del sistema nacional de áreas protegidas del Ecuador, su administración y manejo es diferente: la Reserva Ecológica Antisana está gestionada por el FONAG (Fondo para la Protección del Agua de Quito), y el Ministerio del Ambiente; mientras que el Parque Nacional Cajas está administrado por ETAPA (Empresa de Telecomunicaciones, Agua Potable, Alcantarillado y Saneamiento de Cuenca). Los humedales de este parque fueron reconocidos como sitios RAMSAR por su importancia internacional.

Métodos. La REA y PNC fueron escogidos por la semejanza de sus ecosistemas de páramo (MAE, 2013). En cada área protegida se escogió tres humedales, con diferente estado de conservación, en la 
selección colaboró el personal de cada área protegida. Las zonas para este estudio se eligieron sobre la base de su historia de quema y pastoreo, pero manteniendo características homogéneas de los sitios, como: formación vegetal, cercanía a los centros poblados y rangos de altura. En la Reserva Ecológica Antisana, se identificaron los siguientes humedales: Callejón (conservado), Mica (recuperación) y Callejón Grande (alterado); mientras que, en el Parque Nacional Cajas, fueron escogidos los humedales Tocllacocha (conservado), Tres cruces (recuperación) e Illincocha (alterado).

La contribución de cada humedal a la captura de carbono fue determinada por una muestra constituida por un transecto que atravesaba el bofedal, como lo muestra la Figura 2. En cada transecto se tomaron datos en seis cuadrantes de $0.50 \mathrm{~m}$ x $0.50 \mathrm{~m}$. De cada una de ellos se recopiló la siguiente información para determinar el carbono almacenado:

-Vegetación (Biomasa vegetal): peso verde de vegetación existente en cada cuadrante de $0.50 \mathrm{~m} \times 0.50 \mathrm{~m}$. La muestra para el laboratorio fue de aproximadamente $500 \mathrm{~g}$.

-Sobre el suelo: peso verde de la necromasa y de la que está en proceso de descomposición, la profundidad aproximada de este sutrato. La muestra para el laboratorio fue de aproximadamente $500 \mathrm{~g}$.

-Suelo: se tomaron dos datos a) para el análisis de materia orgánica, una muestra de suelo $500 \mathrm{~g}$ en un rango de profundidad de $0.30 \mathrm{~m}$ en la zona seca y borde, hasta $1.20 \mathrm{~m}$ en la zona más humeda hacia el centro del humedal (Figura 2), b) para la densidad aparente se tomaron dos submuestras de $100 \mathrm{~cm}^{3}$ cada una, utilizando el método del cilindro de volumen conocido (MacDicken, 1997; García \& Cedeño, 2012), con un "muestreador cilíndrico tipo Uhland”.

Para determinar las características químicas del suelo, se tomaron muestras compuestas de cada humedal. En cada cuadrante se tomó una muestra de $100 \mathrm{~cm}^{3}$ de suelo, que fueron homogenizadas en campo en un costal; de la ahí se seleccionó con una pala dos submuestras de 1000 g, para ser llevadas al laboratorio para su análisis.

El análisis de las muestras de suelo, se realizó en el laboratorio AGROCALIDAD, en Quito, y en la Universidad del Azuay, en Cuenca. Las muestras de vegetación, necromasa y suelo fueron secadas a temperatura ambiente en el invernadero, o en la estufa de aire forzado a $50^{\circ} \mathrm{C}$, si presentaban un aspecto muy fangoso. Posteriormente, las muestras fueron molidas y tamizadas a través de un tamiz de $0.002 \mathrm{~m}$ y almacenadas en fundas plásticas transparentes e identificadas, para su posterior análisis. Todos los métodos que se utilizaron para su análisis se sustentan

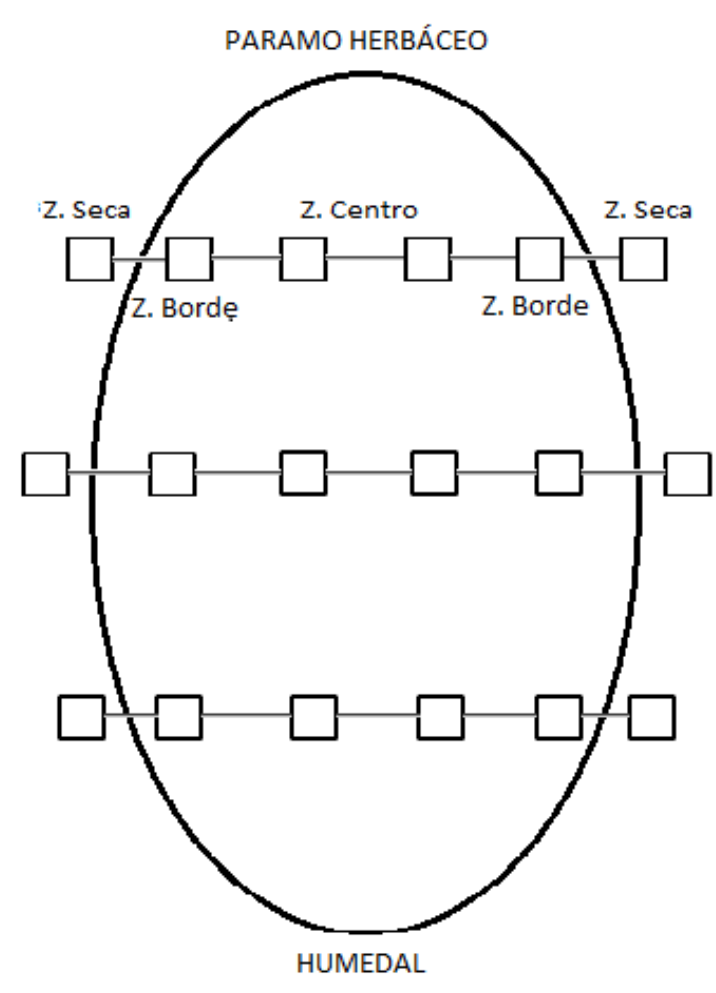

Figura 2. Muestreo a lo largo del humedal.

en métodos estandarizados de la Red de Laboratorios de Suelos del Ecuador (RELASE).

Análisis. Con los resultados de laboratorio, se calculó el carbono orgánico total, que es la suma del carbono de la vegetación (biomasa vegetal), del suelo y sobre el suelo (necromasa), usando las formulas propuestas por Moreno et al. (2002) y Marin Muñiz et al. (2011). Los datos fueron transformados de gramos a mega gramos y de metros a kilómetros, para trabajar con números enteros. Para el análisis se utilizó un arreglo factorial con dos áreas protegidas y tres estados de conservación de los humedales, para el carbono orgánico total y para cada tipo de carbono; en el paquete STATISTICA, se realizó un ANDEVA, la prueba de Tukey para comparación de coeficientes de variación, las comparaciones apareadas $(\mathrm{p} \leq 0.05)$ y el análisis de gráficos estadísticos.

\section{Resultados.}

Características del Suelo

En la Tabla 1 se describen las características químicas de los suelos de los humedales analizados, en la Reserva Ecológica Antisana: Callejón (conservado), Mica (recuperación) y Callejón Grande (alterado); mientras que, en Parque Nacional Cajas, Tocllacocha (conservado), Tres cruces (recuperación) e Illincocha (alterado). 
Tabla 1. Características del suelo de los humedales de la Reserva Ecológica Antisana y el Parque Nacional Cajas.

\begin{tabular}{|c|c|c|c|c|c|c|c|c|c|c|c|c|}
\hline & Humedal & pH & $\begin{array}{l}\text { MO } \\
(\%)\end{array}$ & $\begin{array}{l}N \\
(\%)\end{array}$ & $\begin{array}{c}\mathbf{P} \\
(\mathbf{p p m} \\
\text { ) }\end{array}$ & $\begin{array}{c}\mathrm{K} \\
(\mathrm{cmol} / \mathrm{kg})\end{array}$ & $\begin{array}{c}\mathrm{Ca} \\
(\mathrm{cmol} / \mathrm{kg})\end{array}$ & $\begin{array}{c}\mathrm{Mg} \\
(\mathrm{cmol} / \mathrm{kg})\end{array}$ & $\begin{array}{c}\text { Fe } \\
(\mathbf{p p m})\end{array}$ & $\underset{\text { (ppm) }}{\text { Mn }}$ & $\underset{(\mathrm{ppm})}{\mathrm{Cu}}$ & $\begin{array}{c}\mathrm{Zn} \\
(\mathbf{p p m})\end{array}$ \\
\hline \multirow{6}{*}{$\begin{array}{l}\text { Reserva } \\
\text { Ecológica } \\
\text { Antisana }\end{array}$} & \multirow{2}{*}{$\begin{array}{l}\text { Callejón } \\
\text { Grande }\end{array}$} & 4.8 & 18.5 & 0.9 & 24.5 & 1.2 & 1.7 & 0.9 & 3096.0 & 20.5 & 4.5 & 4.9 \\
\hline & & 5.3 & 5.0 & 0.3 & $<3.5$ & 0.3 & 1.3 & 0.4 & 980.0 & 28.4 & 4.9 & 2.7 \\
\hline & \multirow{2}{*}{ Callejón } & 5.1 & 9.5 & 0.5 & 5.7 & 0.5 & 3.6 & 1.1 & 1382.0 & 29.5 & 12.7 & 6.6 \\
\hline & & 5.3 & 14.7 & 0.7 & $<3.5$ & 0.4 & 6.6 & 1.6 & 728.0 & 66.7 & 7.8 & 11.7 \\
\hline & \multirow{2}{*}{ Mica } & 5.0 & 8.1 & 0.4 & $<3.5$ & 0.2 & 1.9 & 0.9 & 2545.0 & 150.3 & 9.9 & 2.0 \\
\hline & & 4.9 & 7.5 & 0.4 & $<3.5$ & 0.3 & 1.0 & 0.7 & 1394.0 & 20.8 & 9.0 & $<31.6$ \\
\hline \multirow{6}{*}{$\begin{array}{l}\text { Parque } \\
\text { Nacional } \\
\text { Cajas }\end{array}$} & \multirow{3}{*}{ Illincocha } & 5.7 & 7.2 & 0.4 & $<3.5$ & 0.2 & 14.2 & 1.8 & 219.8 & 71.7 & 4.1 & 1.5 \\
\hline & & 6.3 & 29.2 & 1.5 & $<3.5$ & 1.4 & 8.7 & 0.9 & 78.5 & 301.3 & 2.2 & 17.5 \\
\hline & & 5.2 & 27.3 & 1.4 & 13.0 & 0.5 & 7.3 & 0.4 & 2227.0 & 2266 & 6.1 & 12.7 \\
\hline & \multirow{3}{*}{$\begin{array}{l}\text { Tocllacocha } \\
\text { Tres Cruces }\end{array}$} & 4.6 & 9.1 & 0.5 & $<3.5$ & 0.1 & 1.6 & 0.4 & 849.0 & 11.0 & 2.6 & 1.3 \\
\hline & & 4.3 & 56.8 & 2.8 & 45.8 & 1.6 & 17.9 & 1.5 & 258.6 & 236.3 & 0.3 & 28.5 \\
\hline & & 4.7 & 39.0 & 2.0 & 15.0 & 1.3 & 17.9 & 2.0 & 111.0 & 526.0 & 1.6 & 7.2 \\
\hline
\end{tabular}

Los valores encontrados en la REA y PNC (Tabla 1) son característicos para los suelos de páramo del Ecuador (Llambí, et al., 2012). El suelo de la REA es andosol vítrico; mientras que el del Cajas es andosol no alofánico (aluandosol), con una evolución más larga que el andosol vítrico de la REA y puede retener más agua y acumular más carbono, según Podwojewki \& Poulenard (2000 a, b).

Carbono orgánico total

El carbono orgánico total de los humedales de la Reserva Ecológica Antisana tiene una media $683.01+$ $284.64\left(\mathrm{Mg}\right.$ en $0.01 \mathrm{~km}^{2}$ entre 0.30 y $1.20 \mathrm{~m}$ de profundidad) mientras, en el Parque Nacional Cajas la media fue de $320.06 \pm 95.09\left(\mathrm{Mg}\right.$ en $0.01 \mathrm{~km}^{2}$ entre 0.30 y $1.20 \mathrm{~m}$ de profundidad). A pesar de que los humedales seleccionados se encuentran en dos áreas protegidas, con formaciones vegetales similares (MAE, 2013) registran una diferencia significativa en el contenido de carbono orgánico secuestrado en el suelo (Anova F =65.48; p < 0.01; Tabla 2). Estos valores son cercanos a los registrados por Podwojewki \& Poulenard (2000 a, b) en áreas de páramo en el mismo PNC, y en un área cercana a la REA.

En las dos áreas protegidas, el humedal considerado como "conservado" posee el más alto contenido de carbono orgánico (Tabla 2, Figura 3). En el caso de la REA, los tres humedales tienen valores de carbono total son diferentes (Anova $\mathrm{F}=30.36$; $\mathrm{p}<$ 0.01), mientras que en el PNC no existe esta diferencia, pese a la diferencia en el estado de conservación (Anova $\mathrm{F}=0.59 ; \mathrm{p}=0.31$ ).

Carbono en la vegetación

Entre las dos áreas protegidas, el carbono de la vegetación (Biomasa Vegetal), no presenta una diferencia estadísticamente significativa (Anova $\mathrm{F}=0.95 ; \mathrm{p}=0.21$ ), a pesar de que fueron consideradas con estado de conservación heterogéneo por los responsables de las áreas protegidas (Tabla 2, Figura 3). En el caso del PNC, no se presenta diferencia en el contenido de carbono entre los humedales (Anova

Tabla 2. Carbono orgánico almacenado en los humedales de la Reserva Ecológica Antisana y el Parque Nacional Cajas en $0.01 \mathrm{~km}^{2}$.

\begin{tabular}{llccccc}
\hline $\begin{array}{l}\text { Área } \\
\text { Protegida }\end{array}$ & Humedal & Estado & $\begin{array}{c}\text { Carbono } \\
\text { vegetación } \\
\mathbf{( M g}\end{array}$ & $\begin{array}{c}\text { Carbono sobre } \\
\text { suelo } \\
\mathbf{( M g}\end{array}$ & $\begin{array}{c}\text { Carbono suelo } \\
\mathbf{( M g})\end{array}$ & $\begin{array}{c}\text { Contenido Total } \\
\mathbf{( M g )}\end{array}$ \\
\hline $\begin{array}{l}\text { Reserva } \\
\begin{array}{l}\text { Ecológica } \\
\text { Antisana }\end{array}\end{array}$ & Callejón & $\mathrm{C}$ & $196.61 \pm 58.19$ & $504.40 \pm 233.59$ & $55.89 \pm 41.21$ & $794.04 \pm 248.25$ \\
& Mica & $\mathrm{R}$ & $106.9 \pm 54.91$ & $392.84 \pm 238.33$ & $70.65 \pm 30.37$ & $567.56 \pm 258.17$ \\
& Callejón Grande & $\mathrm{A}$ & $168.4 \pm 137.67$ & $394.27 \pm 291.35$ & $42.58 \pm 17.66$ & $687.43 \pm 295.17$ \\
$\begin{array}{l}\text { Parque } \\
\text { Nacional } \\
\text { Cajas }\end{array}$ & Tocllacocha & $\mathrm{C}$ & $136.4 \pm 76.57$ & $44.21 \pm 29.03$ & $151.30 \pm 49.94$ & $334.52 \pm 84.28$ \\
& Tres Cruces & $\mathrm{R}$ & $123.69 \pm 60.90$ & $29.54 \pm 27.69$ & $150.00 \pm 36.44$ & $308.21 \pm 76.46$ \\
& Illincocha & $\mathrm{A}$ & $133.78 \pm 81.62$ & $42.70 \pm 35.39$ & $176.57 \pm 67.99$ & $317.47 \pm 120.87$ \\
\hline
\end{tabular}

Simbología: C (conservado); R (recuperación); A (alterado). 
$\mathrm{F}=1.5$; $\mathrm{p}=0.27$ ), pero en la REA esta diferencia es marcada (Anova $\mathrm{F}=80.03$; $\mathrm{p}<0.01$ ). Esto puede deberse a la diferente historia de manejo del ganado que han tenido en esos ecosistemas. En el caso de Antisana, el ganado fue excluido total o parcialmente de los humedales, y están en mejor proceso de recuperación y conservación; mientras que en Cajas aún se puede encontrar ganado de las comunidades cercanas al área protegida. Esto confirma lo propuesto por Zuñiga-Escobar et al. (2013) que indica: "que los ecosistemas de montaña que se someten a cambio de uso del suelo o disminución de cobertura vegetal para llevar a cabo agricultura o ganadería tienen el peligro que gran parte del carbono se escape a la atmósfera en forma de dióxido de carbono” como sucedió en el PNC.

Además, los restos de vegetación muerta por el ramoneo, según Gallardo \& Merino (2007), “originan metano que es eliminado en forma de gas a la atmósfera”, lo que ocasiona cierta pérdida del carbono capturado por el ecosistema, por esta razón, los datos de carbono del PNC, no tienen diferencia significativa para cada humedal con diferente estado de conservación. Este carbono secuestrado en la vegetación es parte de la producción primaria del humedal, y es el carbono capturado que más fácilmente se pierde por un incendio o ramoneo.

Carbono sobre el suelo

Al analizar el carbono sobre el suelo (el de la necromasa y de aquello en proceso de descomposición) de los distintos estados de conservación de los humedales, no se encontró diferencia significativa para cada área protegida (Tabla 2, Figura 3); pero entre el REA y el PNC sí existe una heterogeneidad en el contenido de total de carbono sobre el suelo (Anova $\mathrm{F}=110.61 ; \mathrm{p}<0.01$ ), siendo menor el contenido de carbono en el PNC. Según, Gallardo \& Merino (2007) "la mayor descomposición de la necromasa, origina un flujo de carbono hacia el suelo", como sucedió en el PNC. Además, como asegura Pérez (1991), "la intervención humana —por ganadería- compacta el suelo y disminuye la porosidad, especialmente en época lluviosa, evitando que la vegetación no puede desarrollarse y se establezcan caminos preferenciales para el escurrimiento del agua”, como sucede en esta zona. Ello ocasiona que gran parte del carbono

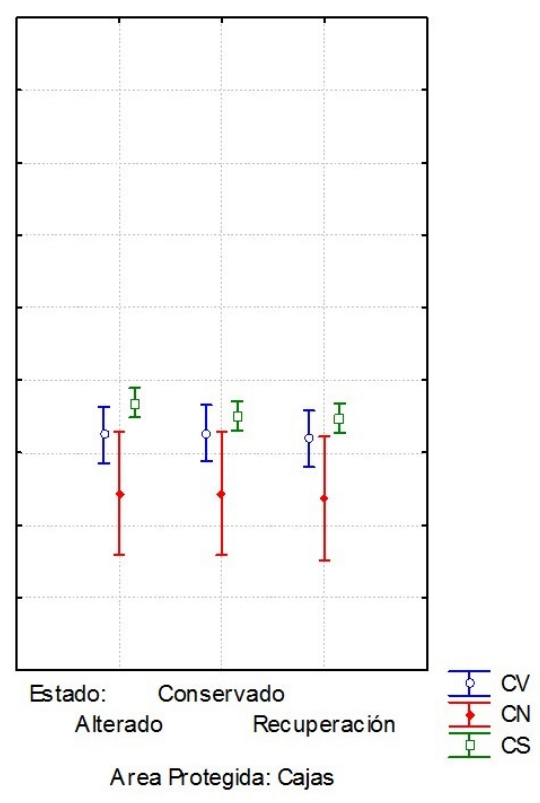
Figura 3. Diferencias de los tipos de carbono orgánico almacenado en la
REA y PNC en $\mathrm{Mg}$ de $\mathrm{C}$ por $0.01 \mathrm{~km}^{2}$ (CV: carbono vegetación, CN: carbono sobre el suelo; CS: carbono del suelo).

almacenado se escape a la atmósfera, en forma de dióxido de carbono.

Carbono orgánico en el suelo

En el suelo, el contenido de carbono es mayor en el PNC que en la REA (Anova $F=65.38$; $p<0.01$ ); pero el contenido de carbono en los humedales para cada área protegida con heterogéneo estado de conservación, no muestra diferencia significativa ( $F=0.87 ; p=0.42$, Tabla 2, Figura 3). La diferencia notable de carbono orgánico en el suelo de las dos áreas protegidas, puede deberse a la presencia de arcilla que tiene el suelo del PNC, ya que mediante una estabilización físico-química de la materia orgánica con partículas arcilla se evita la acelerada descomposición y se mantiene capturado el carbono (Robert, 1996).

El carbono que se encuentra capturado en la vegetación de estos humedales de las áreas protegidas es más vulnerable a regresar a la atmósfera, que el del suelo, ya que por las quemas o ramoneo del ganado en la vegetación de páramo se puede perder hasta el 50 \% del carbono almacenado (Hofstede, 1999; Podwojewski \& Poulenard, 2000b). Estas amenazas son latentes en el sistema nacional de áreas protegidas (SNAP) en el Ecuador (MAE, 2013). Mientras que el carbono en el suelo protegido en las áreas del SNAP, tiene menos probabilidades de regresar a la atmósfera, porque requeriría un impacto drástico en el suelo, como: labranza, secamiento de humedales o sobreuso (Hofstede, 1999; Podwojewski \& Poulenard 2000b), actividades no permitidas por la legislación ecuatoriana dentro de las áreas protegidas del país.

La turba, según Hernández (2010), “se define como el suelo sin consolidar formado por materia 
vegetal fibrosa, parcialmente descompuesta que se acumula en un ambiente anegado". La formación de turba en los humedales representa el sumidero de carbono orgánico más importante en el suelo de estos ecosistemas. En algunos cuadrantes se encontró a 0.60 $m$ de profundidad, casi siempre hacia el centro del humedal. La acumulación de turba ocurre cuando la producción primaria en la superficie excede las pérdidas por descomposición y lixiviados, lo que provoca una acumulación vertical o lateral de depósitos de material orgánico (Hernández, 2010; Turetsky et al., 2004), por eso se encontró cercana a la superficie, en las zonas centrales de los humedales analizados.

Los humedales son considerados importantes sumideros de carbono orgánico, como se ha registrado en la REA y PNC. Al analizarlos en estas dos áreas protegidas, sin embargo, también son fuentes de metano, que es un gas de efecto invernadero (Hernández, 2010), lo que disminuye el posible potencial de sumidero de carbono. Pero en los suelos de los páramos la descomposición es lenta, según Podwojewki \& Poulenard (2000 a, b) “por el régimen de temperatura con promedio bajo y por la formación de complejos aluminio-orgánicos muy estables y parcialmente tóxicos para algunos microorganismos que descomponen la materia orgánica”. La generación de metano $\left(\mathrm{CH}_{4}\right)$ podría ser mínima, pero hay que seguir investigando para resolver las incertidumbres (Bridgham et al., 2006) y conocer el potencial real de carbono secuestrado en los humedales.

Según Jackson (2000), citado por IPCC (2006) lo habitual es que el contenido de C orgánico de los suelos forestales minerales (hasta $1 \mathrm{~m}$ de profundidad) varíe entre 20 y más de $300 \mathrm{Mg}$ de $\mathrm{C}$ en $0.01 \mathrm{~km}^{2}$ según el tipo de bosques y las condiciones climáticas. Por eso, el contenido de carbono en estos humedales de altura es alto en relación a otros ecosistemas boscosos (Aguilar-Arias et al., 2012). Es importante seguir analizando el secuestro de carbono en estos ecosistemas, para que estos puedan ser considerados en estrategias internacionales o nacionales que prevenga su degradación; como la de REDD (Reducción de Emisiones por Deforestación y Degradación Forestal) que se mantiene para ecosistemas boscosos, pero actualmente no incluye a los humedales de altura.

\section{Conclusiones.}

Los humedales tienen un potencial importante de secuestro de carbono, pero el drenado, quemas y pastoreo provocan la oxidación del carbono almacenado en ellos y la liberación de $\mathrm{CO}_{2}$. Estos ecosistemas andinos pueden contribuir a la mitigación de los gases de efecto invernadero, pero es necesario incrementar las investigaciones acerca de la dinámica del carbono, desde que es capturado en la vegetación hasta que pasa a ser parte del suelo.

\section{Agradecimientos.}

El proyecto fue financiado por el Instituto de Transferencia y Tecnología de la Universidad Tecnológica Equinoccial. Además, se agradece el apoyo de los administradores y guardaparques de la REA del Ministerio del Ambiente (MAE), y del personal de ETAPA y el MAE en El Cajas. Agradecemos al Herbario Nacional del Ecuador (QCNE), al laboratorio AGROCALIDAD en Quito, y al de la Universidad del Azuay, en Cuenca, por las facilidades prestadas. Además, a los comentarios de los dos revisores anónimos.

\section{Literatura citada.}

Arias-Aguilar H., Ortiz-Malavassi E., Vilchez-Alvarado B. \& Chazdon R. 2012. Biomasa sobre el suelo y carbono orgánico en el suelo de cuatro estadios de sucesión de bosques en la Península de Osa. Costa Rica. Revista Forestal Mesoamericana, Kurú. Vol 9 No 22. junio 2012

Bridgham S.D., Megonical J.P., Keller J. K., Bliss N.B., \& Trettin C. 2006. The carbon balance of North American wetlands. Wetlands 26:889-916

Busch J. \& Grantham H. 2013. Park versus payments: reconciling divergent policy responses to biodiversity loss and climate change from tropical deforestation. Environmental Research Lettrs 8. (2013) 034028 (10 pp).

Collins M. E. \& Kuehl R. J. 2000. Organic matter accumulation and organic soils. Pp 173-162. En: Richardson J. L. \& J. Vepraskas (Eds.) Wetland soils: genesis, hydrology, landscapes and classification. Lewis Publishers. Boca Raton, FL. USA

Cuesta F., Peralvo M., Baquero F., Bustamante M., Merino A., Muriel P. Freile J. \& Torres O. 2015. Áreas prioritarias para la conservación del Ecuador continental. Ministerio de Ambiente, CONDESAN, Pontificia Universidad Católica del Ecuador, GIZ.

Gallardo J. F. \& Merino A. 2007. El ciclo del carbono y la dinámica de los sistemas forestales. En: Bravo, F. (Coord.). El papel de los bosques españoles en la mitigación del cambio climático. Fundación Gas Natural, Barcelona. 43-64 pp.

Gibbon A., Silman M.R., Malhi Y., Fisher J., Meir P., Zimmermann M. Dargie G.C. Farfan W.R. \& Garcia K. 2010. Ecosystem Carbon Storage Across the Grassland Forest Transition in the High of Manu National Park. Peru. Ecosystems: 15.

Hernández M. 2010. Suelos de humedales como sumideros de carbono y fuentes de metano. Terra Latinoamérica, vol 28, núm 2, abril-junio, pp. 139-147.

Hofstede R. 1999. El páramo como espacio para la fijación de carbono atmosférico. En: Mena, P., C. Josse \& G. Medina (Eds.) 2000. Los Suelos del Páramo. Páramo 5. GTP/Abya Yala. Quito.

.. \& Aguirre N. 1999. Biomasa y dinámica del carbono en relación con las actividades forestales en la Sierra del Ecuador. En: Mena, P., C. Josse \& G. Medina (Eds.) 2000. Los Suelos del Páramo. Páramo 5. GTP/Abya Yala. Quito.

Hossne García A.J. \& Cedeño Campos H.J. 2012. Three methods of determining soil bulk density and solidity in three savanna sandy loam soils. Revista Científica UDO Agrícola, vol. 12, no 4, p. 861-872. 
IPCC (Intergovernmental Panel on Climate Change, UK / US). 2006. Directrices del IPCC de 2006 para los inventarios nacionales de gases de efecto invernadero.

Izurieta X. 2004. Los humedales de altura: Ecosistemas por explorar y proteger. En: Mena, P., \& D. Ortiz (Eds.) 2004. Páramo y Humedales Páramo. Páramo 14. GTP/Abya Yala. Quito.

Llambí L.D., Soto-W A., Célleri R., De Bievre B., Ochoa B. \& Borja P. 2012. Ecología, hidrología y suelos del páramo. Proyecto Páramo Andino.

MAE. 2013. Mapa de vegetación del Ecuador Continental. Quito: Ministerio del Ambiente del Ecuador, Secretaría Nacional de Planificación y Desarrollo, Sistema Nacional de Información.

MacDicken K. 1997. A Guide to Monitoring Carbon Storage in Forestry and Agroforestry Projects. Forest Carbon Monitoring Program.

Mitsch W. J. \& Gosselink J.G. 2000. Wetlands. Tercera Edición. John Wiley and Son. New York, NY. USA

Marín Muñiz J., Hernández Alarcón M.E. \& MorenoCasasola Barceló P. 2011. Secuestro de carbono en suelos humedales costeros de agua dulce en Veracruz. Tropical and Subtropical Agroecosystems, vol. 13, núm. 3, 2011, pp. 365-372

Moreno E., Guerrero A., Gutiérrez M., Ortiz C. \& Palma D. 2002. Los manglares de Tabasco, una reserva natural de carbono. Madera y Bosques. 8: 115-128.

Podwojewski P. \& Poulenard J. 2000a. Los Suelos de los páramos del Ecuador. En: Mena, P., C. Josse \& G.
Medina (Eds.) 2000. Los Suelos del Páramo. Páramo 5. GTP/Abya Yala. Quito.

2000 b.La degradación de los suelos en los páramos. En: Mena, P., C. Josse \& G. Medina (Eds.) 2000. Los Suelos del Páramo. Páramo 5. GTP/Abya Yala. Quito.

Pérez F.L. 1991. Particle sorting due to the off-road vehicle traffic in a high Andean páramo. Catena. 18:239-254.

Rangel Ch. 2000. Colombia Diversidad Biótica III La región de vida paramuna. Universidad Nacional de ColombiaInstituto de Ciencias Naturales, Instituto de Investigación en Recursos Biológicos Alexander von Humboldt.

Robert M. 1996. Le sol: interface dansl'environnement.Ressource pour le développement. Dunod/Masson. Paris 240 pp.

Turetsky M.R, Mannig S.W. \& Wieder R. 2004. Dating recent peat deposits. Wetlands 24: 324-356.

Vega E. \& Martinez D. 2000. Productos Económicamente Sustentables y Servicios Ambientales de Páramo. Serie Paramo 4. GTP/Abya Yala. Quito.

Zúñiga-Escobar O., Peña-Salamanca E., Torres-González A., Cuero-Guependo R. \& Peña-Óspina J. 2013. Assessment of the impact of anthropic activities on carbon storage in soils of high montane ecosystems in Colombia. Agronomía Colombiana, vol. 31, núm. 1, enero-abril, 2013, pp. 112-119 Colombia.

\footnotetext{
${ }^{1}$ Docente. Dirección de Posgrado, Universidad Tecnológica Equinoccial, Quito-Ecuador. david.suarez.duque@gmail.com

${ }^{2}$ Maestría en Turismo y Áreas Protegidas. Dirección de Posgrado, Universidad Tecnológica Equinoccial, QuitoEcuador

${ }^{3}$ Docente. Carrera de Administración de Empresas Turísticas y Conservación Ambiental, Universidad Tecnológica Equinoccial, Quito-Ecuador
} 